\title{
openheart MicroRNA 8059 as a marker for the presence and extent of coronary artery calcification
}

\author{
Philippa Howlett, ${ }^{1}$ Jane K Cleal, ${ }^{2}$ Huihai Wu, ${ }^{1}$ Nikunj Shah, ${ }^{1}$ Alex Horton, ${ }^{3}$ \\ Nick Curzen, ${ }^{4,5}$ Michael Mahmoudi ${ }^{4,5}$
}

To cite: Howlett P, Cleal JK, Wu $\mathrm{H}$, et al. MicroRNA 8059 as a marker for the presence and extent of coronary artery calcification. Open Heart 2018;5:e000678. doi:10.1136/ openhrt-2017-000678

Received 14 June 2017 Revised 14 November 2017 Accepted 11 December 2017
D Check for updates

${ }^{1}$ Department of Cardiology, University of Surrey, Guildford, UK

${ }^{2}$ Institute of Developmental Sciences, University of Southampton, Southampton, UK ${ }^{3}$ Cardiology, Royal Surrey County Hospital, Guildford, UK

${ }^{4}$ Wessex Cardiac Unit, University Hospital Southampton NHS

Foundation Trust, Southampton, UK

${ }^{5}$ Faculty of Medicine, University of Southampton, Southampton, UK

Correspondence to Dr Michael Mahmoudi; $\mathrm{m}$. mahmoudi@soton.ac.uk

\section{ABSTRACT}

Objective MicroRNAs (miRNAs) may serve as potential biomarkers in a variety of pathologies. The aim of this study was to determine whether miRNAs could serve as blood-based markers of isolated coronary artery calcification (CAC) defined as CAC in the absence of an underlying metabolic abnormality.

Methods 24 age-matched and sex-matched patients who had been referred for elective CT coronary calcium score and angiography as part of investigation for cardiac chest pain were recruited. Peripheral venesection was performed and an Agatston calcium score was derived from the CT coronary angiogram using default software. RNA was extracted using the LeukoLOCK Total RNA Isolation System for Toray's microarray analysis and quantitative reverse transcription PCR (qRT-PCR).

Results The patients were well matched for age, sex and conventional risk factors for coronary artery disease. Microarray analysis identified lower expression of miRNA-138-2-3p, miRNA-1181, miRNA-6816-3p and miRNA-8059 in patients with coronary artery calcium score $(C A C S)=0$ vs CACS $>100$. qRT-PCR confirmed significant downregulation of miRNA-8059 in patients with CACS $>100$ (CACS $=0$ vs CACS $>100 ; P=0.03$ ).

Conclusion miRNA-8059 may serve as a peripheral blood-based biomarker for the presence of CAC, as well as provide a platform for studying the pathophysiological basis of isolated CAC.

Trial registration number NCT01992848; Results.

\section{BACKGROUND}

Atherosclerotic coronary artery disease (CAD) remains the most common cause of morbidity and mortality in the Western world. ${ }^{1}$ In approximately $50 \%$ of individuals, the initial presentation of CAD is a myocardial infarction or sudden cardiac death. As a consequence, international guidelines such as the American Heart Association and the European Society of Cardiology have recommended the use of screening tests to identify high-risk individuals. ${ }^{23}$ However, conventional clinic-based models such as the Framingham Risk Score, which creates three categories of 10 -year risk for a future cardiovascular event, can only predict $60 \%-65 \%$ of individuals at

\section{Key questions}

What is already known about this subject? The role of microRNAs (miRNAs) in patients with coronary artery calcification (CAC) has not been examined.

What does this study add? We have identified miRNA 8059 to be significantly downregulated in patients with extensive CAC.

How might this impact on clinical practice? miRNA 8059 may serve as a novel biomarker for the presence and extent of CAC, as well as provide a platform to study the molecular mechanism underpinning vascular calcification.

risk of a cardiovascular event, and are therefore unable to identify many individuals who go on to have an acute cardiac event. ${ }^{4}$ Recent studies have focused on the assessment of the degree of coronary artery calcification (CAC) as a risk assessment tool for cardiovascular events. The clinical utility of coronary CT for the assessment of CAC and the presence of obstructive CAD has been well validated, but its applicability may be limited by cost, poor image quality in patients with obesity and its relative contraindication in patients with renal impairment and contrast allergy. ${ }^{5-8}$ MicroRNAs (miRNAs) are short, non-coding RNAs that regulate gene expression by annealing to partially complementary sites on the 3' untranslated region of messenger RNA (mRNA) to suppress their translation. ${ }^{9}$ A number of studies have demonstrated the clinical utility of miRNAs in the diagnosis and prognosis of a number of cardiovascular disorders such as myocardial infarction, stable angina and heart failure. ${ }^{10-12}$ Furthermore, recent studies have also identified miRNAs to play a role in arterial calcification; for example, miRNA-204 and miRNA-125b have been implicated in vascular smooth muscle calcification in in vitro models. ${ }^{13} 14$ As 


\begin{tabular}{|c|c|c|c|c|c|}
\hline Variable & $\mathrm{CAC}=0(n=6)$ & CAC $=1-10(n=6)$ & CAC $=11-100(n=6)$ & CAC $>100(n=6)$ & $P$ value \\
\hline Age (years) & $55.7 \pm 5.8$ & $51.3 \pm 9.0$ & $53.4 \pm 7.9$ & $54.0 \pm 6.4$ & 0.80 \\
\hline Male sex & $4(66.7 \%)$ & $3(50 \%)$ & $4(66.7 \%)$ & $5(83.3 \%)$ & 0.70 \\
\hline $\operatorname{BMI}\left(\mathrm{kg} / \mathrm{m}^{2}\right)$ & $24.8 \pm 4.3$ & $25.1 \pm 2.3$ & $27.6 \pm 4.8$ & $25.3 \pm 2.2$ & 0.69 \\
\hline Hypertension & $3(50 \%)$ & $2(33.3 \%)$ & $2(33.3 \%)$ & $1(16.7 \%)$ & 0.70 \\
\hline Dyslipidaemia & $1(16.7 \%)$ & $1(16.7 \%)$ & $2(33.3 \%)$ & $3(50 \%)$ & 0.55 \\
\hline Current smokers & $4(66.7 \%)$ & $2(33.3 \%)$ & $2(33.3 \%)$ & $3(50 \%)$ & 0.73 \\
\hline FHx for CAD & $0(0 \%)$ & $3(50 \%)$ & $3(50 \%)$ & $3(50 \%)$ & 0.20 \\
\hline Haemoglobin (g/L) & $14.6 \pm 0.89$ & $14.4 \pm 1.4$ & $15.3 \pm 1.1$ & $15.3 \pm 1.4$ & 0.35 \\
\hline Creatinine ( $\mu \mathrm{mol} / \mathrm{L})$ & $74.7 \pm 17.7$ & $74.8 \pm 15.1$ & $73.2 \pm 8.5$ & $78.2 \pm 7.0$ & 0.86 \\
\hline Total cholesterol (mmol/L) & $5.5 \pm 0.70$ & $5.0 \pm 0.8$ & $5.6 \pm 0.7$ & $5.7 \pm 1.1$ & 0.45 \\
\hline Triglycerides (mmol/L) & $1.1 \pm 0.3$ & $1.2 \pm 0.6$ & $1.8 \pm 0.8$ & $1.8 \pm 1.1$ & 0.19 \\
\hline Glucose (mmol/L) & $5.0 \pm 1.5$ & $4.9 \pm 0.6$ & $5.0 \pm 0.4$ & $5.2 \pm 0.4$ & 0.94 \\
\hline Corrected calcium (mmol/L) & $2.2 \pm 0.1$ & $2.2 \pm 0.1$ & $2.3 \pm 0.1$ & $2.3 \pm 0.0$ & 0.09 \\
\hline \multicolumn{6}{|l|}{ Medication } \\
\hline Calcium channel antagonists & $2(33.3 \%)$ & $2(33.3 \%)$ & $0(0 \%)$ & $0(0 \%)$ & 0.21 \\
\hline ACE inhibitors & $1(16.7 \%)$ & $0(0 \%)$ & $0(0 \%)$ & $1(16.7 \%)$ & 0.67 \\
\hline Statins & $0(0 \%)$ & $0(0 \%)$ & $0(0 \%)$ & $2(33.3 \%)$ & 0.89 \\
\hline Aspirin & $0(0 \%)$ & $0(0 \%)$ & $0(0 \%)$ & 2 (33.3\%) & 0.89 \\
\hline Diuretics & $0(0 \%)$ & $0(0 \%)$ & $1(16.7 \%)$ & $0(0 \%)$ & 0.41 \\
\hline
\end{tabular}

$\mathrm{BMI}$, body mass index; CAC, coronary calcium score; CAD, coronary artery disease; FHx, family history.

a consequence, identification of an 'miRNA signature' that is characteristic of CAC may represent a novel, safe and non-invasive approach in the diagnosis of patients with CAC.

This study was designed to determine whether human blood-based miRNAs could represent plausible biomarkers for the presence and extent of CAC in patients with isolated CAC (defined as CAC in the absence of an underlying metabolic abnormality known to be associated with CAC), which could then be used as a platform to study the molecular mechanism(s) responsible for isolated CAC.

\section{METHODS}

\section{Patients}

The miRNAs as Potential Biomarkers for Coronary Artery Calcification (MAP-Calcification) was a prospective study

\begin{tabular}{lccc} 
Table 2 & CT-derived data for the four cohorts of patients \\
\hline Variable & $\begin{array}{l}\text { Calcium } \\
\text { score }\end{array}$ & $\begin{array}{l}\text { Percentile } \\
\text { for age }\end{array}$ & $\begin{array}{l}\text { Number of } \\
\text { diseased vessels }\end{array}$ \\
\hline CAC $=0$ & $\begin{array}{l}\text { Not } \\
\text { Applicable }\end{array}$ & Not Applicable & Not Applicable \\
CAC $=1-10$ & $1.7 \pm 1.0$ & $50 \pm 28.6$ & $0.2 \pm 0.4$ \\
CAC $=11-100$ & $39.5 \pm 29.7$ & $54.7 \pm 29.9$ & $0.3 \pm 0.5$ \\
CAC $>100$ & $278 \pm 20.5$ & $83.7 \pm 17$ & $2.2 \pm 1.3$ \\
\hline
\end{tabular}

CAC, coronary calcium score. registered at ClinicalTrials.gov (NCT01992848). All participants gave informed consent before enrolment. Twenty-four patients who were matched for sex, age, hypertension and smoking history undergoing elective CT calcium scoring and coronary angiography as part of routine clinical investigation for cardiac chest pain were prospectively recruited. Clinical inclusion criteria were age $\geq 18$ years and ability to provide written informed consent. Exclusion criteria were age $>65$ years, diabetes mellitus, glomerular filtration rate $\leq 60 \mathrm{~mL} / \mathrm{min}$, abnormal serum calcium, history or clinical features of cardiac failure, valvular heart disease, previously documented CAD, history of coronary revascularisation, history of cerebrovascular disease, peripheral vascular disease, systemic inflammatory disease, active infection and active malignancy.

\section{CT coronary calcium scoring}

Coronary artery calcium scores (CACS) were obtained using a General Electric (GE) VCT XTe 64-slice CT scanner. All patients had an unenhanced cardiac-gated calcium score calculated using the GE Advantage Workstation 4.4 with the default software. An optimal heart rate (aiming for $<60$ beats $/ \mathrm{min}$ ) was achieved with the administration of variable doses of metoprolol. Of the $150 \mathrm{~mL}$ of Niopam 370 contrast, $150 \mathrm{~mL}$ were injected at $6 \mathrm{~mL} / \mathrm{s}$, followed by $50 \mathrm{~mL}$ of normal saline solution at $6 \mathrm{~mL} / \mathrm{s}$. Subsequent CT coronary angiography was performed with prospective cardiac-gated acquisition. 

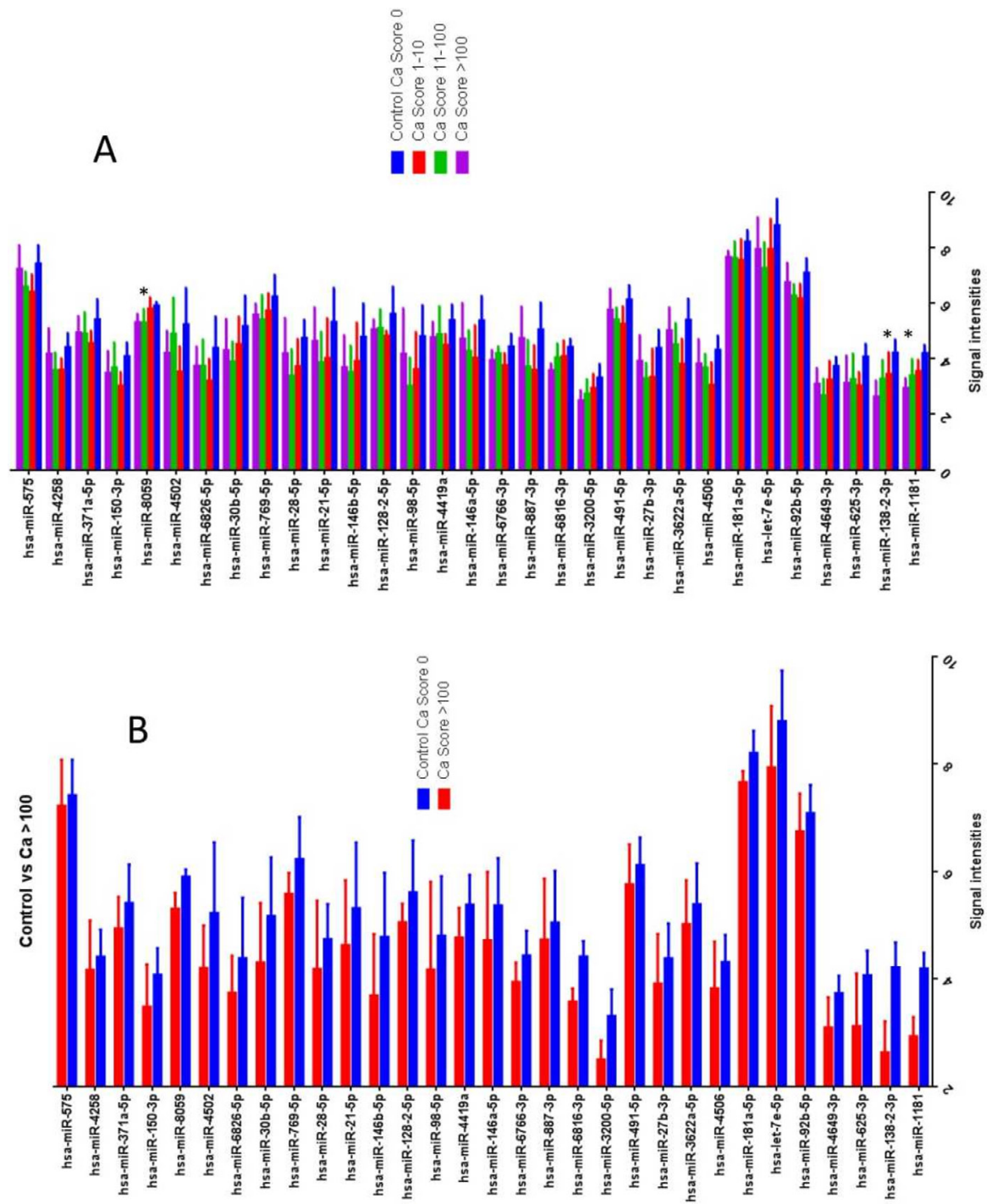

Figure 1 Toray microarray analysis of the four groups of patients $(A)$ and in patients with calcium score $>100$ (B). These data indicate that the expression of four microRNAs is significantly reduced in patients with calcium score $>100$ compared with controls: miRNA-138-2-3p, miRNA-1181, miRNA-6816-3p and miRNA-8059. Data are presented as mean \pm SEM. Ca, calcium.

\section{Blood collection and analysis}

Venous blood samples were drawn via antecubital venepuncture. Routine haematology and biochemistry were assessed in plasma. An aliquot of whole blood $(10 \mathrm{~mL})$ for each subject was collected and total RNA isolated using the LeukoLOCK Total RNA Isolation System with DNase treatment, following manufacturer's instructions (Thermo Fisher Scientific, Warrington, UK). Total RNA quantity and quality were determined using a NanoDrop Technologies ND-1000 spectrophotometer and an Agilent 2100 Bioanalyzer. All samples had an RNA integrity number greater than 7 . Toray microarray analysis of all the known human miRNAs and the relevant bioinformatics analysis of the 24 patients were undertaken at the Cambridge Genomic Centre, UK.

Quantitative reverse transcription PCR (qRT-PCR) of four miRNAs (miRNA-8059, miRNA-138-2 and miRNA1181) that were identified on bioinformatic analysis of the microarray data was performed using the Qiagen
miScript system, following manufacturer's instructions (Qiagen, Crawley, UK). Briefly, total RNA (250 ng) underwent reverse transcription using the miScript II RT Kit with HiSpec Buffer to convert the mature miRNAs into complementary DNA. Specific miRNAs were purified using the SYBR Green PCR Kit with specific miScript primer assays. The cycle parameters were $95^{\circ} \mathrm{C}$ for $15 \mathrm{~min}$, followed by 40 cycles of $95^{\circ} \mathrm{C}$ for $15 \mathrm{~s}, 55^{\circ} \mathrm{C}$ for $30 \mathrm{~s}$ and $70^{\circ} \mathrm{C}$ for $35 \mathrm{~s}$. All samples were analysed in duplicates. The geometric mean of RNU-6 and SNORD 61 was used to normalise the miRNA expression data. There was an agreement between qRT-PCR values and a mean value of duplicates was used. The coefficient of variation of the qRT-PCR assay was $<10 \%$. Pathway analysis for the mRNA targets for the differentially expressed miRNAs was carried out using DIANA-miRPath V.3 in order to determine the possible signalling cascades that the identified miRNAs may be regulating. ${ }^{15}$ 


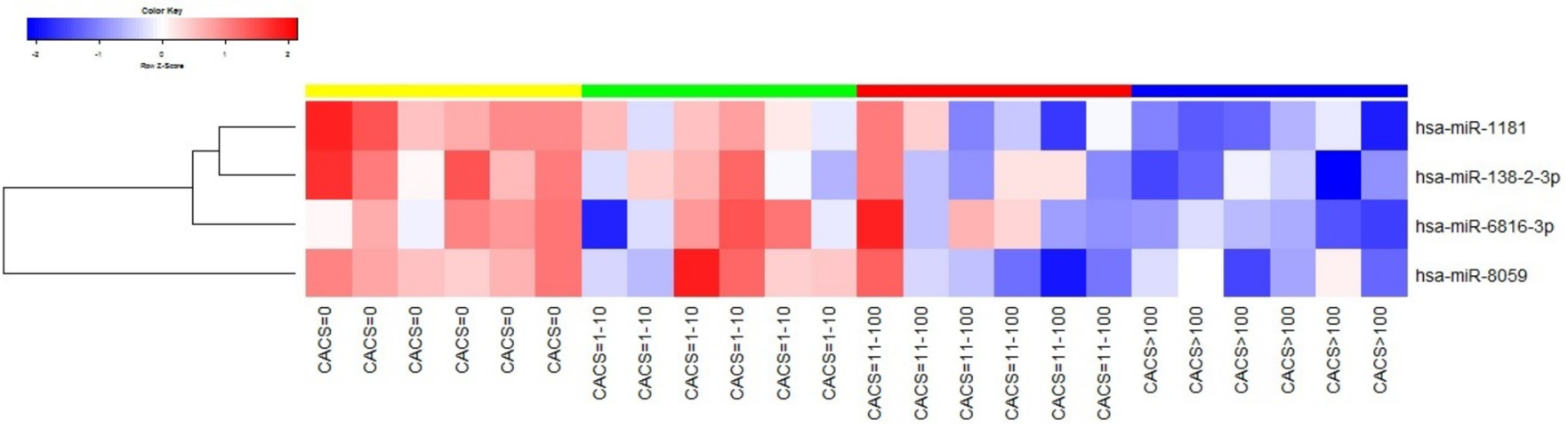

Figure 2 Heat map of microRNA microarray expression from the four groups of patients. The expression range 'lowmoderate-high' is indicated by the colour range 'blue-white-red'. The upper colour bar represents the groups as follows: yellow for $\mathrm{CACS}=0$, green for $\mathrm{CACS}=1-10$, red for $\mathrm{CACS}=11-100$ and blue for CACS $>100$. CACS, coronary artery calcium score.

\section{Statistical analysis}

A formal power calculation was not undertaken as this was a proof-of-concept study. Statistical analysis was performed using SAS V.8.2. Continuous variables and categorical variables are expressed as mean $\pm \mathrm{SD}$, number of patients and percentages, respectively. Clinical demographics, CT variables, microarray and qRT-PCR data were analysed using a one-way analysis of variance or two-tailed unpaired t-test with Welch correction as appropriate. A P value $<0.05$ was considered statistically significant.

\section{RESULTS}

The patients were divided into four groups based on their calcium score in order to determine whether differences in miRNA were attributable to subtle differences in the CACS: $\mathrm{CACS}=0, \mathrm{CACS}=1-10, \mathrm{CACS}=11-100$ and CACS $>100$. The four groups of patients were well matched for age, sex, body mass index, hypertension, dyslipidaemia, current smoking habit and family history for CAD (table 1). The patients also had similar haemoglobin, creatinine, total cholesterol and triglyceride, glucose, and corrected calcium concentrations (table 1). There were differences in the medical therapy across the four groups of patients (table 1).

The mean calcium score was $1.7 \pm 1.0$ in patients with CACS $=1-10,39.5 \pm 29.7$ in patients with $\mathrm{CACS}=11-100$, and $278 \pm 20.5$ in patients with CACS $>100$ (table 2). The number of diseased vessels was $0.2 \pm 0.4$ in patients with CACS $=1-10,0.3 \pm 0.5$ in patients with $\mathrm{CACS}=11-100$, and $2.2 \pm 1.3$ in patients with CACS $>100$ (table 2 ).

Toray microarray analysis of the known human miRNAs and subsequent bioinformatics analysis of the generated data indicated significant downregulation of miRNAs 138-2-3p, 1181, 6816 and 8059 in patients with CACS $>100$

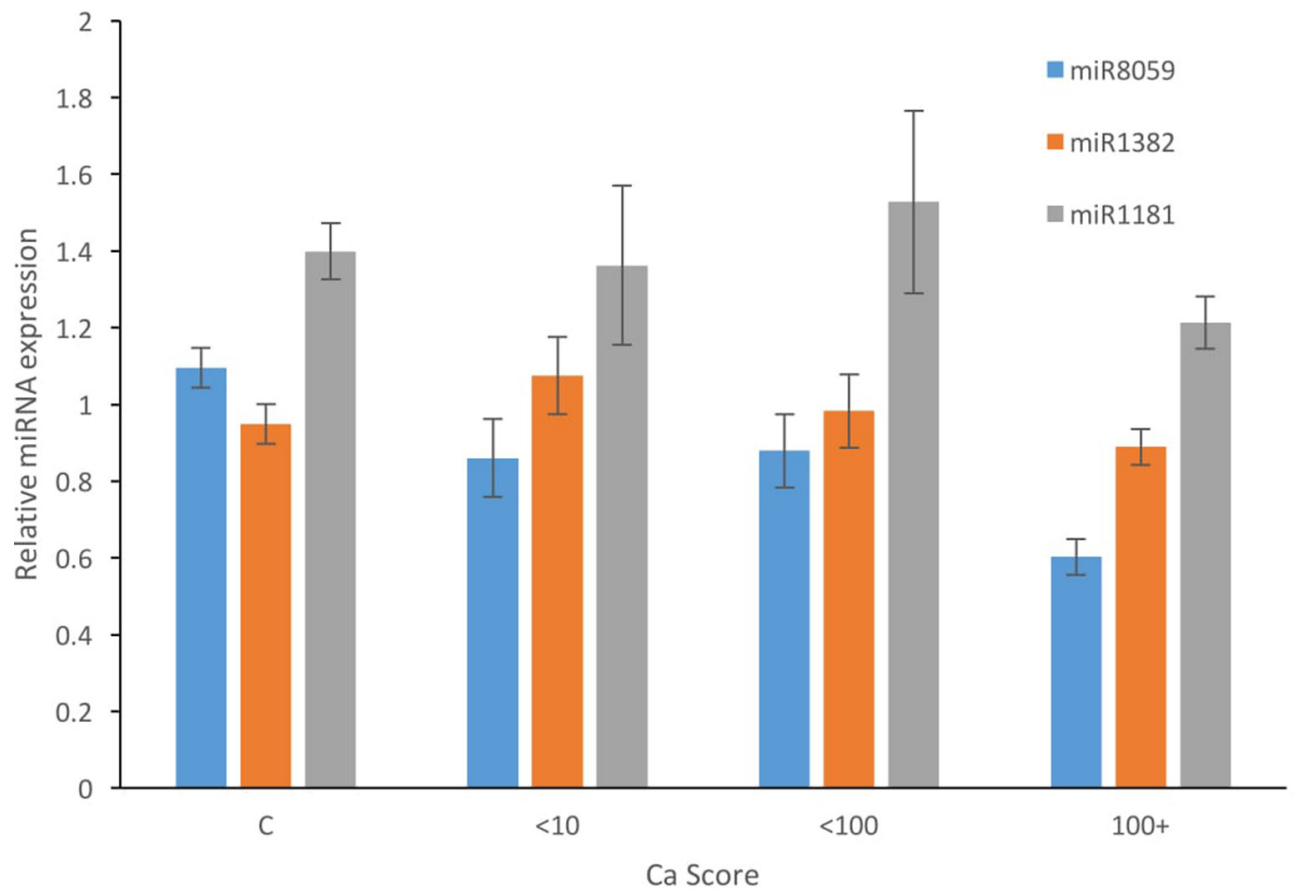

Figure 3 Quantitative reverse transcription PCR analysis indicated significant downregulation of miR-8059 in patients with coronary artery calcification score $>100(P=0.03)$. Ca, calcium. 
Table 3 Gene pathways predicted to be regulated by miRNA-8059

\begin{tabular}{llll}
\hline KEGG pathway & P value & $\begin{array}{l}\text { Genes } \\
\text { (n) }\end{array}$ & $\begin{array}{l}\text { miRNAs } \\
\text { (n) }\end{array}$ \\
\hline $\begin{array}{l}\text { Metabolism of xenobiotics by } \\
\text { cytochrome P450 }\end{array}$ & $1.34 \mathrm{E}-20$ & 2 & 1 \\
$\begin{array}{l}\text { Biosynthesis of unsaturated fatty } \\
\text { acids }\end{array}$ & $6.66 \mathrm{E}-11$ & 1 & 1 \\
\hline $\begin{array}{l}\text { Fatty acid metabolism } \\
\text { Chemical carcinogenesis }\end{array}$ & $3.44 \mathrm{E}-06$ & 2 & 1 \\
\hline $\begin{array}{l}\text { Glycosaminoglycan biosynthesis- } \\
\text { keratan sulfate }\end{array}$ & 0.0023 & 2 & 1 \\
\hline $\begin{array}{l}\text { Glycosaminoglycan degradation } \\
\text { Nucleotide excision repair }\end{array}$ & 0.013 & 1 & 1 \\
\hline $\begin{array}{l}\text { Glycosphingolipid biosynthesis } \\
\text { Proteoglycans in cancer }\end{array}$ & 0.013 & 2 & 1 \\
\hline
\end{tabular}

KEGG, Kyoto encyclopedia of genes and genomes.

as compared with patients with $\mathrm{CAC}=0$ (figures 1 and 2). qRT-PCR analysis confirmed significant downregulation of only miRNA-8059 in patients with CACS $>100$ (figure 3). Pathway analysis was carried out using the online tool DIANA Tools miRPath, and this identified the mRNA pathways targeted by miRNA- $8059^{15}$ (table 3 ).

\section{DISCUSSION}

In this study, we investigated the association between peripheral circulating miRNAs and extent of CAC in patients with isolated CAC. Expression profiling identified downregulation of four miRNAs, with miRNA-8059 being validated using qRT-PCR. Specifically, miRNA-8059 was shown to be significantly downregulated in patients with $\mathrm{CAC}>100$. Our results suggest that miRNA-8059 has the potential to serve as blood-based biomarker for the presence and extent of CAC.

Recent studies have focused on the assessment of the degree of CAC as a robust risk assessment tool. CAC has indeed been shown to be an independent predictor of mortality, in a multivariate model controlling for age, sex, ethnicity and cardiac risk factors. ${ }^{16}$ Risk-adjusted relative risk ratios for CAC were 2.2-fold, 4.5-fold, 6.4-fold, 9.2-fold, 10.4-fold and 12.5-fold for scores of 11-100, 101-299, 300-399, 400-699, 700-999 and >1000when compared with a score of 0 . Although vascular calcification is most commonly associated with chronic kidney disease and diabetes mellitus, CAC may occur in isolation in patients with coronary atherosclerosis. The close correlation between the extent of CAC and the atherosclerotic burden has been confirmed by histopathological and intravascular studies so that the CACS, as measured by CT coronary angiography, has been established as an anatomical surrogate of overall cardiac plaque burden. ${ }^{17}$ CT coronary angiography is, however, of limited use as a screening modality due to its availability, cost and radiation exposure.

miRNAs have emerged as robust diagnostic and prognostic markers in a wide range of clinical conditions ranging from oncology, immunology and cardiovascular disorders. ${ }^{19} 20$ The mechanism(s) underlying isolated CAC remains largely elusive. It is established that chronic inflammation within the plaque microenvironment is an important factor in the pathogenesis of coronary calcification. Specifically, for example, macrophages, lymphocytes and dendritic cells within the plaque secrete cytokines such as tumour necrosis factor that can activate the Msx2-Wnt signalling leading to augmentation of nuclear B-catenin accumulation and osteogenic differentiation. ${ }^{21}$ The pro-oxidant, $\mathrm{H}_{2} \mathrm{O}_{2}$, has also been shown to promote osteochondrocytic differentiation of vascular smooth muscle cells through upregulation of the osteogenic transcription factor Runx2. ${ }^{22}$ Products of lipid peroxidation such as oxidised-LDL (low density lipoprotein) also induce osteogenic-mediated and apoptosis-mediated calcifications of vascular cells. ${ }^{23}$ Calcification may also be induced by a variety of metabolic factors such as pyrophosphate and urate crystals, vitamin $\mathrm{D}$, as well as glucose. ${ }^{24}$ miRNA- 8059 has been implicated in the regulation of many of the genes that contribute to the above processes, including Uridine diphosphate-N-acetylglucoeamine (UDP-GLCNAc), involved in lymphocyte trafficking; vitamin D receptor, bone morphogenetic protein receptor, involved in bone and cartilage formation; apolipoprotein L, involved in cholesterol transport; and mitochondrial calcium uptake 1 , which prevents mitochondrial calcium overload, thus suppressing the production of reactive oxygen species. Given the diverse influence of miRNA-8059 on such pathways, we speculate that miRNA-8059 may represent a novel blood-based biomarker for the presence of CAC, as well as provide a platform for studying the potential mechanism(s) that may underline CAC.

The role of miRNAs in human CAC has not been thoroughly investigated. Liu et $a l^{25}$ analysed plasma miRNA levels of 11 patients with CACS $>100$ and 6 matched patients with $\mathrm{CACS}=0$ and demonstrated eight miRNAs (223, 3135b, 133a-3p, 2861, 134, 191-3p, 3679-5p, 1229) that were significantly increased in patients with CAC and four miRNAs (2861, 134, 1229 and 3135b) that were correlated with the degree of CAC. A number of significant differences may account for the variations between our results and those of Liu et al. First, the focus of our study was on isolated CAC, and for this reason we had devised a strict exclusion criteria excluding, among others, patients with diabetes mellitus, peripheral vascular disease and metabolic abnormalities known to be associated with CAC. By contrast, Liu et al had much broader inclusion criteria, including $23.4 \%$ of their population being non-insulin-requiring patients with diabetes. Second, we studied miRNAs in the leucocyte population as opposed to the plasma, which may have accounted for the observed differences. Finally, whether 
ethnic variations (Caucasian vs Chinese) have any impact on miRNA expression has not been previously addressed.

This study has several limitations. We isolated miRNAs from peripheral leucocytes rather than microvesicles, which are known to contain the vast majority of miRNAs, and this may have influenced our results. Furthermore, we have not correlated whether systemic alterations in the expression of miRNA-8059 are associated with similar alterations at the level of coronary arteries.

In conclusion, miRNA-8059 is downregulated in the blood of patients with CACS $>100$ and may serve as a biomarker for the presence of isolated CAC, as well as provide a platform to study the underlying pathophysiology of CAC.

Contributors $\mathrm{PH}, \mathrm{NS}, \mathrm{NC}$ and MM were involved in devising the project, analysing the data and writing the manuscript. PH and NS were also responsible for blood collection and RNA preparation. JKC undertook all the PCR work. HW was responsible for bioinformatic data analysis. AH undertook the CT investigations and reporting. All authors reviewed the manuscript prior to submission.

Funding This research received no specific grant from any funding agency in the public, commercial or not-for-profit sectors.

Competing interests None declared.

Patient consent Obtained.

Ethics approval Surrey Borders Research Ethics Services Committee (REC reference: 13/L0/0644; IRAS Project ID: 123526).

Provenance and peer review Not commissioned; externally peer reviewed.

Data sharing statement Data is available from the corresponding author upon reasonable request.

Open Access This is an Open Access article distributed in accordance with the Creative Commons Attribution Non Commercial (CC BY-NC 4.0) license, which permits others to distribute, remix, adapt, build upon this work non-commercially, and license their derivative works on different terms, provided the original work is properly cited and the use is non-commercial. See: http://creativecommons.org/ licenses/by-nc/4.0/

(c) Article author(s) (or their employer(s) unless otherwise stated in the text of the article) 2018. All rights reserved. No commercial use is permitted unless otherwise expressly granted.

\section{REFERENCES}

1. British Heart Foundation. Heart Statistics. http://www.bhf.org.uk/ research/heart-statistics

2. Greenland P, Alpert JS, Beller GA, et al. 2010 ACCF/AHA guideline for assessment of cardiovascular risk in asymptomatic adults: executive summary: a report of the American College of Cardiology Foundation/American Heart Association Task Force on Practice Guidelines. Circulation 2010;122:2748-64.

3. Piepoli MF, Hoes AW, Agewall S, et al. 2016 European Guidelines on cardiovascular disease prevention in clinical practice: The Sixth Joint Task Force of the European Society of Cardiology and Other Societies on Cardiovascular Disease Prevention in Clinical Practice (constituted by representatives of 10 societies and by invited experts) Developed with the special contribution of the European Association for Cardiovascular Prevention \& Rehabilitation (EACPR). Eur Heart J 2016;37:2315-81.
4. Raggi P, Cooil B, Shaw LJ, et al. Progression of coronary calcium on serial electron beam tomographic scanning is greater in patients with future myocardial infarction. Am J Cardiol 2003;92:827-9.

5. Agatston AS, Janowitz WR, Hildner FJ, et al. Quantification of coronary artery calcium using ultrafast computed tomography. J Am Coll Cardiol 1990;15:827-32.

6. Schuleri KH, George RT, Lardo AC. Applications of cardiac multidetector CT beyond coronary angiography. Nat Rev Cardiol 2009;6:699-710.

7. Detrano R, Hsiai T, Wang S, et al. Prognostic value of coronary calcification and angiographic stenoses in patients undergoing coronary angiography. J Am Coll Cardiol 1996;27:285-90.

8. Wong ND, Hsu JC, Detrano RC, et al. Coronary artery calcium evaluation by electron beam computed tomography and its relation to new cardiovascular events. Am J Cardiol 2000;86:495-8.

9. Wightman B, Bürglin TR, Gatto J, et al. Negative regulatory sequences in the lin-14 3'-untranslated region are necessary to generate a temporal switch during Caenorhabditis elegans development. Genes Dev 1991;5:1813-24.

10. Wang GK, Zhu JQ, Zhang JT, et al. Circulating microRNA: a novel potential biomarker for early diagnosis of acute myocardial infarction in humans. Eur Heart J 2010;31:659-66.

11. D'Alessandra $Y$, Devanna $P$, Limana $F$, et al. Circulating microRNAs are new and sensitive biomarkers of myocardial infarction. Eur Heart J 2010;31:2765-73.

12. Taurino C, Miller WH, McBride MW, et al. Gene expression profiling in whole blood of patients with coronary artery disease. Clin Sci 2010;119:335-43.

13. Cui RR, Li SJ, Liu LJ, et al. MicroRNA-204 regulates vascular smooth muscle cell calcification in vitro and in vivo. Cardiovasc Res 2012;96:320-9.

14. Goettsch C, Rauner M, Pacyna N, et al. miR-125b regulates calcification of vascular smooth muscle cells. Am J Pathol 2011:179:1594-600.

15. Vlachos I, Zagganas K, Paraskevopoulou M, et al. DIANA-mirPath v3.0: deciphering microRNA function with experimental support. Nucl Acids Res 2015.

16. Budoff MJ, Shaw LJ, Liu ST, et al. Long-term prognosis associated with coronary calcification: observations from a registry of 25,253 patients. J Am Coll Cardiol 2007;49:1860-70.

17. Mintz GS, Pichard AD, Popma JJ, et al. Determinants and correlates of target lesion calcium in coronary artery disease: a clinical, angiographic and intravascular ultrasound study. J Am Coll Cardiol 1997;29:268-74.

18. Baumgart D, Schmermund A, Goerge G, et al. Comparison of electron beam computed tomography with intracoronary ultrasound and coronary angiography for detection of coronary atherosclerosis. J Am Coll Cardiol 1997;30:57-64.

19. Lu J, Getz G, Miska EA, et al. MicroRNA expression profiles classify human cancers. Nature 2005;435:834-8.

20. Rosenfeld N, Aharonov R, Meiri E, et al. MicroRNAs accurately identify cancer tissue origin. Nat Biotechnol 2008;26:462-9.

21. Al-Aly Z, Shao JS, Lai CF, et al. Aortic Msx2-Wnt calcification cascade is regulated by TNF-alpha-dependent signals in diabetic Ldlr-/- mice. Arterioscler Thromb Vasc Biol 2007;27:2589-96.

22. Byon $\mathrm{CH}$, Javed $\mathrm{A}$, Dai $\mathrm{Q}$, et al. Oxidative stress induces vascular calcification through modulation of the osteogenic transcription factor Runx2 by AKT signaling. J Biol Chem 2008;283:15319-27.

23. Nadra I, Mason JC, Philippidis P, et al. Proinflammatory activation of macrophages by basic calcium phosphate crystals via protein kinase $C$ and MAP kinase pathways: a vicious cycle of inflammation and arterial calcification? Circ Res 2005;96:1248-56.

24. Price PA, June HH, Buckley JR, et al. Osteoprotegerin inhibits artery calcification induced by warfarin and by vitamin $\mathrm{D}$. Arterioscler Thromb Vasc Biol 2001;21:1610-6.

25. Liu W, Ling S, Sun W, et al. Circulating microRNAs correlated with the level of coronary artery calcification in symptomatic patients. Sci Rep 2015;5:16099. 